Subclinical Leishmania infection in patients with rheumatic diseases under biological drugs

Martina Maritati, Alessandro Trentini, Gregory Michel, Tiziana Bellini, Shawgi Almugadam, Stefania Hanau, Marcello Govoni, et al.

\section{Infection}

A Journal of Infectious Diseases

ISSN 0300-8126

Infection

DOI 10.1007/s15010-018-1189-2

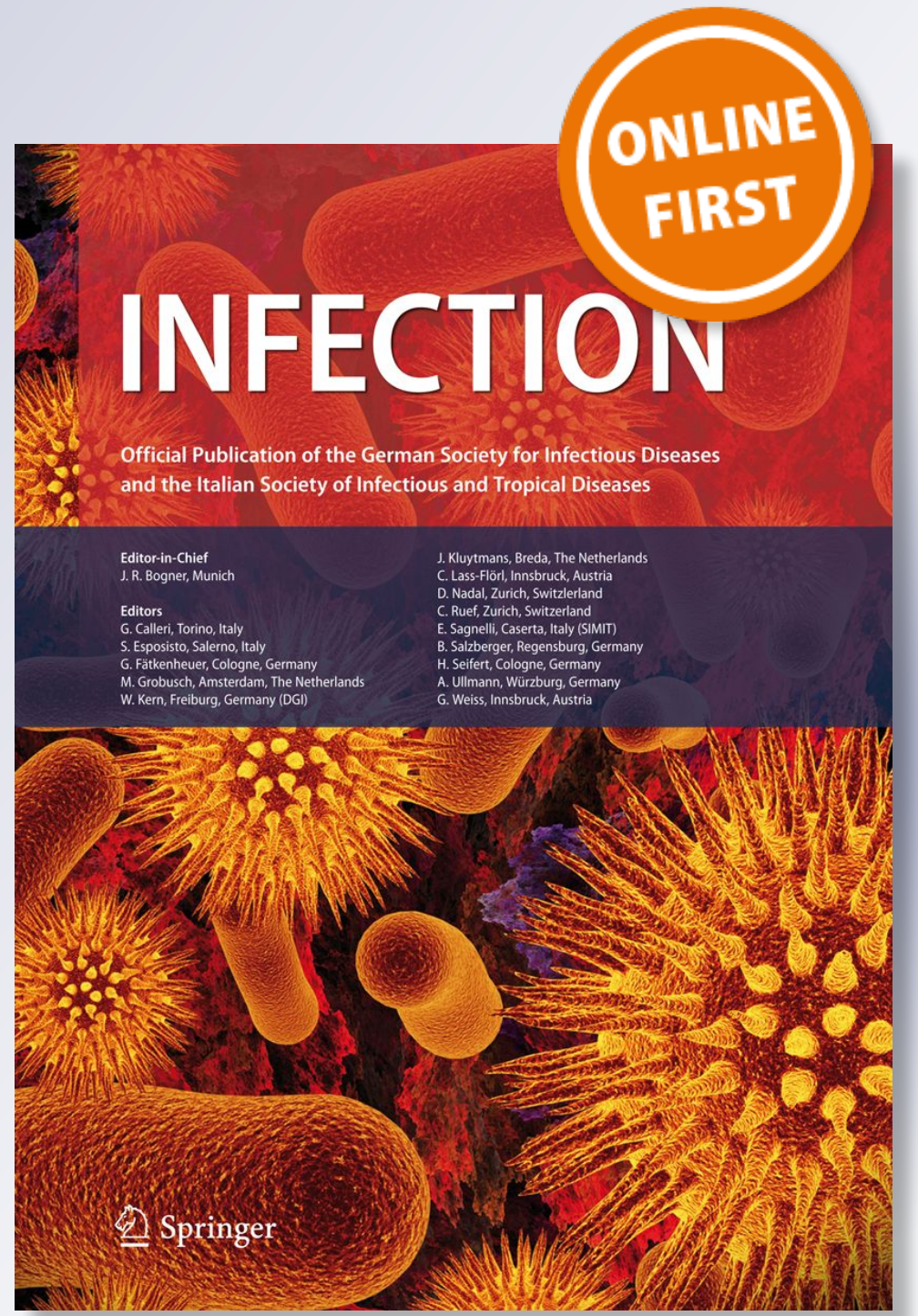

黛 Springer 
Your article is protected by copyright and all rights are held exclusively by SpringerVerlag GmbH Germany, part of Springer Nature. This e-offprint is for personal use only and shall not be self-archived in electronic repositories. If you wish to self-archive your article, please use the accepted manuscript version for posting on your own website. You may further deposit the accepted manuscript version in any repository, provided it is only made publicly available 12 months after official publication or later and provided acknowledgement is given to the original source of publication and a link is inserted to the published article on Springer's website. The link must be accompanied by the following text: "The final publication is available at link.springer.com". 


\title{
Subclinical Leishmania infection in patients with rheumatic diseases under biological drugs
}

\author{
Martina Maritati ${ }^{1}$ - Alessandro Trentini ${ }^{2} \cdot$ Gregory Michel $^{3,4} \cdot$ Tiziana Bellini $^{2} \cdot$ Shawgi Almugadam $^{2}$. \\ Stefania Hanau ${ }^{2} \cdot$ Marcello Govoni $^{6} \cdot$ Pierre Marty $^{3,4,5} \cdot$ Carlo Contini $^{1}$ (D)
}

Received: 20 April 2018 / Accepted: 31 July 2018

○) Springer-Verlag GmbH Germany, part of Springer Nature 2018

\begin{abstract}
Purpose Climate changes and immunosuppression are influencing the spread of leishmaniasis and re-emergence in Northern Italy, respectively. We evaluated the prevalence of subclinical leishmaniasis in patients from a Northern Italian region with chronic inflammatory rheumatism (CIRD) receiving biological drugs (BD) and correlated it to the area of residence.

Methods DNA from PBMC of patients affected by CIRD treated with either BD for at least 5 years (Group A) or other immunosuppressive drugs (Group B) was investigated by a qPCR for Leishmania infantum kDNA and compared to healthy subjects (Group C). Variables such as sex and age, rural areas, dog ownership, type of BD administered and association between $\mathrm{BD}$ and steroids, were evaluated by statistical analysis.

Results A higher proportion of L. infantum DNA positivity was found in Group A than in Group C $(p<0.05)$, while no parasite DNA was detected in Group B. In Group A, 18/50 patients (36\%) had higher rates of parasite DNA (from 1 to 136 to 1.000 .000 copies $/ \mathrm{ml}$ ) than Group C (from 1 to 10 copies $/ \mathrm{ml}$ ). 14/18 (77.7\%) of positive patients from Group A lived in rural areas, but no statistical differences occurred in relation to dog ownership or BD type $(p<0.0003)$.

Conclusions We can speculate that exposure to rural areas appears to be a factor closely linked with the risk of developing Leishmania subclinical infection. A screening with molecular methods in patients with CIRD treated with BD living in these areas and monitoring Leishmania DNA during such therapies, would be mandatory to prevent delay in diagnosis should VL symptoms appear.
\end{abstract}

Keywords Leishmania infantum · Subclinical leishmaniasis · Biologic drugs $\cdot$ Rural areas $\cdot$ Northern Italy $\cdot$ Real-time PCR

Carlo Contini

cnc@unife.it

1 Section of Infectious Diseases and Dermatology, Department of Medical Sciences, University of Ferrara, 44124 Ferrara, Italy

2 Section of Medical Biochemistry, Molecular Biology and Genetics, Department of Biomedical and Specialist Surgical Sciences, University of Ferrara, Ferrara, Italy

3 INSERM, U1065, Centre Méditerranéen de Médecine Moléculaire, C3M, Toxines Microbiennes dans la Relation Hôte-Pathogènes, 06204 Nice, France

4 Faculté de Médecine, Université de Nice Sophia Antipolis, Nice, France

5 Parasitologie-Mycologie, Centre Hospitalier Universitaire l'Archet, Université Côte d'Azur, CS 23079, 06202 Nice, France

6 Section of Hematology and Rheumatology, Department of Medical Sciences, University of Ferrara, Ferrara, Italy
Abbreviations

VL Visceral leishmaniasis

CL Cutaneous leishmaniasis

CIRD Chronic inflammatory rheumatic diseases

RA Rheumatoid arthritis

PA Psoriatic arthritis

AS Ankylosing spondylitis

BD Biological drugs

qPCR Quantitative real-time PCR

\section{Background}

Leishmaniasis is one of the most important human-neglected parasitic diseases and is caused by Leishmania species transmitted by insect bites. Approximately, 12 million individuals are currently infected with Leishmania spp. with an estimated 2 million new cases occurring every year [1]. The major endemic areas are in the tropics and subtropics; 
however, leishmaniasis exclusively due to Leishmania infantum is also endemic in large parts of Southern Europe where there is a focal prevalence of latent infection of up to $53 \%$ in the adult population [2], but it is a rare disease in other parts of Europe or North and Central Europe where it is diagnosed almost exclusively in travellers or migrants coming from tropical or subtropical countries [3]. In the world, several Leishmania spp., in particular L. donovani/infantum complex, constitute the predominant group as the ones responsible for causing substantial health problems in up to 400,000 people and 40,000 deaths per year [1, 4, 5].

In Italy, visceral leishmaniasis (VL) the most serious form of the disease to be distinguished from the more common cutaneous leishmaniasis (CL), is an endemic disease present in rural and peri-urban areas of continental and insular regions of Central and South Italy [6]. About 40-70 cases are notified annually, over half of which are from Sicily (data from the Italian Institute of Statistics, ISTAT).

In Emilia-Romagna, a Northern region of Italy, 44 cases were diagnosed in the period 1999-2011 of which 26 were VL; the number of cases rose to 30 in 2013 and the regional capital Bologna, was the most affected city [7].

Although environmental aspects such as climate change, growing urbanisation and socio-economic development are causing important changes in the epidemiology of some infectious diseases including leishmaniasis [8], host immunity is known to represent a key determinant of the clinical manifestation and outcome of this disease. In particular, immunosuppression has been identified as a major contributor to disease re-emergence in Europe other than in AIDS patients, in those with autoimmune rheumatic diseases who live in endemic areas $[4,9]$.

In this setting, a number of published case reports suggest an association between TNF- $\alpha$ antagonist use and evident leishmaniasis [10, 11].

One aspect not yet fully defined concerns the start of TNF- $\alpha$ antagonist therapy in patients with autoimmune diseases that require treatment with these drugs, already suffering from a $L$. infantum subclinical infection.

In these last years, the availability of polymerase chain reaction (PCR) assays amplifying leishmanial genetic material, has been a major step forward in improving the diagnosis of leishmanial disease and the response to treatment compared to serological methods which may fail to detect asymptomatic Leishmania infections especially in endemic areas [12]. These techniques have also been successfully used to detect follow-up of VL patients [13] and subclinical infections [14-16] and were suggested as marker of $L$. infantum infection in asymptomatic individuals for epidemiological studies in India [13].

Our study aimed to evaluate by a real-time PCR (qPCR) the prevalence of subclinical leishmaniasis in immunocompetent individuals living in some rural and suburban areas of
Emilia-Romagna region suffering from inflammatory rheumatic diseases (CIRD) receiving immunosuppressive treatment with biological drugs (BD) (Group A). This prevalence was compared to that found in two control groups consisting of rheumatologic patients who did not consider biological therapies and healthy individuals (B and C), respectively, matched for age and geographical area according to previous preliminary results [17]. Genomic positivity was related to the area of residence within the region, the dog ownership and, for Group A, to the type of BD administered and the concomitant association with steroids.

\section{Materials and methods}

\section{Study patients and design}

We performed a retrospective observational study enrolling 50 patients affected by CIRDs (rheumatoid arthritis, psoriatic arthritis and ankylosing spondylitis) treated with $\mathrm{BD}$ for at least 5 years at the Rheumatology Unit of the University of Ferrara (Group A) and two control groups consisting of 50 patients affected by CIRDs treated with drugs other than BD (Group B) and 50 healthy subjects (Group C). The three cohorts were selected from the same regional area.

Demographic and clinical data were collected using standard questionnaires, which included the region of provenance, the province of residence, the location of their home in an urban or rural context, and the ownership of a dog as a family pet.

All the enrolled subjects were negative by serologic screening for hepatitis B and C, HIV and tuberculosis and did not show any symptoms at the moment of the blood sampling. The CD4/CD8 ratio was $>2$ in all the enrolled subjects.

\section{Blood sample collection, PBMC preparation and DNA extraction}

The isolation of PBMC was done from $10 \mathrm{ml}$ of EDTA-anticoagulated blood by density gradient centrifugation over Ficoll-paque plus (Amersham Biosciences Europe Gmbh, Milan, Italy), according to the manufacturer's protocol. DNA was extracted from PBMC within $2 \mathrm{~h}$ after collection, as previously described [18]. Briefly, each sample was added to an equal volume of lysis buffer containing proteinase $\mathrm{K}$ (200 mg/ml), incubated for $1 \mathrm{~h}$ at $56^{\circ} \mathrm{C}$ and then heated for 10 min at $96{ }^{\circ}$ C. L. infantum DNA was extracted by phenol-chloroform-isoamyl alcohol followed by ethanol precipitation, and resuspended in TE buffer. Reference DNA extracted from Leishmania promastigotes cultures was provided. Promastigotes of canine L. infantum MON-1 (provided by IZSLER, Modena, Italy) were routinely cultured 
in RPMI 1640 medium (pH 7.2) (Sigma, Germany) supplemented with $15 \%$ heat inactivated fetal calf serum (HIFCS) (Gibco, Paisley, UK), $100 \mathrm{U} / \mathrm{ml}$ penicillin, and $100 \mathrm{U} / \mathrm{ml}$ streptomycin sulphate in $25 \mathrm{~cm}^{2}$ non-vented flasks (Corning, USA) [19]. Parasites were then incubated at $25^{\circ} \mathrm{C}$ in a humidified incubator under air as a gas phase with medium replacement every 3-4 days. DNA preparations extracted from clinical blood specimens originating from patients with confirmed VL were also included in this study as positive control. In general, one blood sample was collected for each subject/patient.

\section{Real-time PCR}

A real-time PCR (qPCR) for detection and quantification of L. infantum DNA was performed according to Lachaud et al. [20]. For accurate sensitivity, kinetoplast DNA was chosen as the molecular target. External primers were derived from RV1 and RV2 as previously described [21]. DNA detection was performed by means of a hybridization probe based on TaqMan chemistry (Applera, Courtaboeuf, France) designed with Primer 3 software. A Stratagene (La Jolla, Calif.) MX 4000 system was used for amplification and detection. Optimization experiments led us to use the Stratagene qPCR master mix (catalog number 600549-51), 15 pmol of forward primer (CTTTTCTGGTCC TCCGGGTAGG), 15 pmol of reverse primer (CCACCCGGCCCTATTTTAC ACCAA), and 50 pmol of TaqMan probe (FAM-TTTTCGCAGAAC GCCCCT ACCCGC-TAMRA). Assays were performed with a $25 \mu \mathrm{l}$ final volume with $1 \mu \mathrm{l}$ of sample DNA. Two negative controls (DNA extracted from buffy coat obtained from a Leishmania-seronegative individual and an aliquot of distilled water, respectively) were included in each PCR run to detect contamination which could lead to false-positive results. The standard curve was established from Leishmania DNA extracted from $5 \times 10^{6}$ parasites; $1 \mu$ of serial dilutions, ranging from the equivalent of 50,000 to 0.0001 parasites, was introduced into reaction tubes. TaqMan chemistry allowed two-step temperature ( 94 and $55^{\circ} \mathrm{C}$ ) cycling over 45 cycles. Linearity of quantification was made by serial dilution from 10 (4) to $10^{-1}$ dilution. Quantification was given in parasite per $\mathrm{ml}$. Within each run, linearity was checked with two dilutions 10 and $10^{-1}$, according to Mary et al. [22]. All qPCR reactions were performed at ParasitologyMycology Laboratory of the Archet Hospital, University of Nice, France.

\section{Statistical analysis}

Data analysis was performed using SPSS Statistics for Windows, version 21.0 (SPSS, Inc., Chicago, IL, USA). Normality of distribution for continuous variable was checked by Shapiro-Wilk test. Since the variables were not normally distributed, group comparisons between controls and patients affected by CIRDs were performed using Mann-Whitney $U$ test.

Chi-square tests were used to compare differences in categorical variables. To assess the association of the PCR positivity to Leishmania (outcome variable) with explanatory variables such as living in a rural area, having a dog or taking a steroid in association with a biological drug, a binary logistic regression analysis in the positive subgroup of CIRDs subjects was performed. To avoid possible overcorrection of the regression models, three different logistic regressions were performed, one for each explanatory variable, including age and sex as confounding factors in each model. Two-tailed probability values $<0.05$ were considered statistically significant.

\section{Ethical considerations}

The study was approved by the ethics committee of the University of Ferrara, Italy. Written informed consents were obtained from all participants before their enrolment.

\section{Results}

Demographical and clinical characteristics of the study population $(n=150)$ are summarized in Table 1. Group A patients were older than those from Group C $(p<0.001)$ without any difference with Group B; moreover, no significant difference in sex distribution between the three groups was found. There were no statistically significant differences between $\mathrm{A}, \mathrm{B}$ and $\mathrm{C}$ groups regarding staying in a rural area and owning a dog as a family pet. Finally, Group A showed a statistically significant positivity for $L$. infantum circulant kDNA compared to Group C $(p<0.001)$, but not with Group $\mathrm{B}$, since none of the subjects in this group was found to be

Table 1 Demographical characteristics of rheumatologic patients receiving $\mathrm{BD}(\mathrm{A})$, rheumatologic patients receiving drugs other than $\mathrm{BD}(\mathrm{B})$ and healthy controls (C)

\begin{tabular}{llll}
\hline Patient's characteristics & $\mathrm{A}(n=50)$ & $\mathrm{B}(n=50)$ & $\mathrm{C}(n=50)$ \\
\hline Age (years) & $61(49-67)^{\mathrm{a}}$ & $57(34-72)$ & $55(31-60)$ \\
Gender (females, \%) & 70 & 62 & 70 \\
Rural area (\%) & 36 & 38 & 36 \\
To be owner of a dog (\%) & 36 & 38 & 34 \\
Positivity for circulant & $36^{\mathrm{b}}$ & 0 & 10 \\
$\quad$ Leishmania DNA (\%) & & & \\
\hline
\end{tabular}

Not-normally distributed variables are expressed as median (interquartile range), discrete variables as percentage

${ }^{\mathrm{a}} p<0.001$, Mann-Whitney vs. Group C

${ }^{\mathrm{b}} p<0.001, \chi^{2}$ vs. Group C; the Group B was excluded from statistical analyses due to proportion equal to zero 
positive and thus was excluded from the analysis. The three cohorts of patients were then analysed separately.

\section{Group A patients}

Of the 50 analysed samples from Group A, 18 (36\%; 14 women and 4 men, median age of 58.7), resulted positive by qPCR with the detection of a high parasite burden (1-136 parasites/ml in 4 patients, 1,000-40,000 in 11 patients and over 1,000,000 in 3 patients).

Laboratory features regarding Leishmania DNA detected by qPCR analysis and correlation with rural residence are summarized in Table 2.

Group A patients were than divided into negative and positive according to the detection of Leishmania DNA (Table 3).

All positive patients presented a long history of rheumatic disease begun between 8 and 29 years, when patients experienced symptoms, with a mean value of $20.3 \pm 5.6$. Of these, $12 / 18(77.8 \%)$ suffered from rheumatoid arthritis (RA), 3/18 (11.1\%) from ankylosing spondylitis (AS) and psoriatic arthritis (PA). 14/18 (66.6\%) lived in rural areas as defined by the Organization for Cooperation and Economic Development (OECD; http://www.oecd.org/regional/). To have a dog did not influence the positivity to Leishmania.
Table 3 Negative and positive patients from Group A according to detection of Leishmania DNA by qPCR analysis

\begin{tabular}{lll}
\hline Group A & qPCR & \\
\hline Patient's characteristics & Negative $(n=32)$ & Positive $(n=18)$ \\
Age (years) & $58.4 \pm 13.2$ & $58.9 \pm 9.5$ \\
Disease duration (years) & $22.0 \pm 5.7$ & $20.3 \pm 5.6$ \\
Therapy duration (years) & $10.7 \pm 2.7$ & $10.6 \pm 3.4$ \\
Rural area (\%, yes) & 21.8 & $66.6^{\mathrm{a}}$ \\
To be owner of a dog (\%, yes) & 31.3 & 50.0 \\
Rheumatologic disease (\%) & & \\
RA & 61.7 & 77.8 \\
PA & 6.0 & 11.1 \\
AS & 32.3 & 11.1 \\
BD treatment (\%) & & \\
Anti-TNF- $\alpha$ & 81.4 & 55.6 \\
T Lymphocyte modulator & 9.3 & 22.2 \\
Anti-IL-6 receptor & 9.3 & 22.2 \\
Steroid drug (\%, yes) & 25.0 & $61.1^{\mathrm{b}}$ \\
\hline
\end{tabular}

Continue variables are expressed as mean $\pm \mathrm{SD}$, discrete variables as percentage. RA: rheumatoid arthritis

$P A$ psoriatic arthritis, $A S$ ankylosing spondylitis

${ }^{\mathrm{a}} p<0.001, \chi^{2}$ vs. negative

${ }^{\mathrm{b}} p<0.05 \chi^{2}$ vs. negative
Table 2 L. infantum kDNA load (copies/ml) in Group A and Group C and correlation with rural residence

\begin{tabular}{|c|c|c|c|}
\hline $\begin{array}{l}\text { Membership group of the } \\
\text { studied population }\end{array}$ & $\begin{array}{l}\text { No. of rheumatologic patients positive } \\
\text { to } L \text {. infantum circulant DNA }\end{array}$ & Rural areas & $\begin{array}{l}\text { Parasite DNA } \\
\text { load (copies/ } \\
\mathrm{ml} \text { ) }\end{array}$ \\
\hline \multirow[t]{18}{*}{ Group A } & 1 & No & 136 \\
\hline & 2 & Yes & 21.666 \\
\hline & 3 & Yes & 27.266 \\
\hline & 4 & No & 15.000 \\
\hline & 5 & Yes & 5.956 \\
\hline & 6 & No & 0.2 \\
\hline & 7 & Yes & 28.066 \\
\hline & 8 & Yes & 24.366 \\
\hline & 9 & Yes & 39.400 \\
\hline & 10 & Yes & 6.5 \\
\hline & 11 & No & 1.650 \\
\hline & 12 & Yes & 1.960 .000 \\
\hline & 13 & Yes & 3.056 \\
\hline & 14 & Yes & 1.286 .666 \\
\hline & 15 & Yes & 2.423 .000 \\
\hline & 16 & Yes & 7.610 \\
\hline & 17 & Yes & 100 \\
\hline & 18 & Yes & 100 \\
\hline \multirow[t]{4}{*}{ Group C } & 1 & Yes & 10 \\
\hline & 2 & Yes & 1 \\
\hline & 3 & Yes & 1 \\
\hline & 4 & Yes & 1 \\
\hline
\end{tabular}


In Table 3 patients positive to $L$. infantum DNA are grouped, according to the pharmacological action of the BD into TNF- $\alpha$ antagonists, modulators of T lymphocyte activity and anti-IL-6 receptor antibodies. The possible influence of each type of drug on the molecular positivity for L. infantum circulant kDNA was assessed through a Chi-square test. No statistical difference among the different administered treatments $\left[\chi^{2}(2)=3.37, p=0.185\right]$ was found, although the percentage of positive subjects to qPCR was higher when under therapy with modulators of T lymphocyte activity and anti-IL-6 receptor antibodies compared to TNF- $\alpha$ antagonist therapy $(57.1 \%, 50.0 \%$ and $26.3 \%$, respectively). A further statistical analysis was performed on the basis of the concurrent, or not, administration of steroids together with the BD.

Interestingly, patients that were taking a steroid in association with the BD showed a higher proportion of Leishmania DNA positivity than those assuming the BD only $\left[\chi^{2}(1)=6.18, p<0.05 ; 55.6 \%\right.$ vs. $\left.21.2 \%\right]$. Finally, by binary logistic regression we evaluated whether the kind of living area, to be owner of a dog or taking a steroid drug were associated to an increased risk of being positive to molecular method. As shown in Table 4, the fact of living in a rural area or taking a steroid associated to the assumption of a biological therapy, were factors strongly linked to an increased risk of being positive to $L$. infantum kDNA [O.R. (95\%CI): 8.74 (2.24-34.05) and 5.52 (1.4021.78), respectively], independently from confounding factors such as sex and age.

\section{Patients from Group B and C}

No sample among those analysed from Group B was positive by qPCR. In the Group C, 4 positive (8\%) samples were detected by qPCR (10 parasites/ml in 1 patient and 1 parasite/ml in 3 patients) (Table 2). Of the healthy subjects who resulted to be positive, only one owned a dog.

\section{Discussion}

Global warming and man-made risk factors such as travel, migration, trade of animals and environmental modifications, are changing the temporal-spatial evolution of leishmaniasis in Europe which is hypoendemic especially in Mediterranean countries and remains in most cases asymptomatic, although immunosuppression can predispose to clinical disease [23-25].

In Northern Italy, leishmaniasis has been spreading in part because of climatic conditions that favour the development of the vector Phlebotomus perniciosus [26, 27], but also for the increased mobility of dogs to and from the traditional VL endemic areas [27], although the risk for human disease does exist but it is moderate, as the intensity of transmission seems to be lower than in traditional settings of Mediterranean VL.

Moreover, immunosuppressive drugs, in particular BD, have contributed to expand the number of reported cases in this area. In fact, the commercialization of the BD (such as TNF- $\alpha$ antagonists) has become increasingly frequent since the end of the 1990s and has been proven to be

Table 4 Binary logistic regression for the risk of being positive to Leishmania DNA in subjects living in rural areas, having a dog, and taking steroid drugs in association with the BD

\begin{tabular}{lll}
\hline & $\mathrm{B}(\mathrm{SE})$ & Odds ratio $(95 \% \mathrm{CI})$ \\
\hline Model 1: rural area & & \\
Intercept & $-2.52(2.00)$ & $8.74(2.24-34.05)$ \\
Rural area (yes/no) & $2.18(0.69)^{* *}$ & \\
Model 2: to be owner of a dog & $-1.952(1.78)$ & $2.58(0.73-9.18)$ \\
Intercept & $0.948(0.65)$ & \\
Dog (yes/no) & $-0.27(1.64)$ & $5.53(1.40-21.78)$ \\
Model 3: steroids & $1.71(0.70)^{*}$ & \\
Intercept &
\end{tabular}

For Model 1: $R^{2}=0.199$ (Cox \& Snell), 0.278 (Nagelkerke)

For Model 2: $R^{2}=0.044$ (Cox \& Snell), 0.061 (Nagelkerke)

For Model 3: $R^{2}=0.121$ (Cox \& Snell), 0.168 (Nagelkerke)

B: unstandardized regression coefficient; SE: standard error; 95\% CI: 95\% confidence interval

Both models were corrected for possible confounders including age and sex, with living in a rural area, having a dog and assumption of steroid drugs in association with the biological therapy as predictors, and positivity to Leishmania as dependent variable

$* p<0.05, * * p<0.001$ 
effective in the management of several immune diseases, including CIRDs, RA, AS and psoriasis [28]. Their use, however, is not free of side effects especially for the onset of opportunistic infections, including leishmaniasis [29].

We aimed to investigate asymptomatic $L$. infantum human infection in Emilia-Romagna, an Italian region not traditionally considered as an area of endemicity, characterized by continental climate and by a low incidence of officially reported VL cases, but recently switched to "phlebotomus risk region" because of repeated and uninterrupted contacts with the vector and the animal reservoirs, as some coastal and hilly regions of the middle Adriatic and those of pre-Alpine Northern Italy. Canine leishmaniasis, has indeed been increasing in Northern Italy, spreading to rural and peri-urban areas as recently demonstrated [27].

Our preliminary results have demonstrated a strikingly higher prevalence of parasite DNA in Group A than in Group B and C ( $p<0.001, \chi^{2}$ vs. controls) in a sample consisting of 50 patients with CIRD (Group A) treated with BD for at least 5 years compared to 50 rheumatologic patients assuming drugs other than BD (Group B) and 50 healthy controls randomized from the apparently health population (Group C). A large percentage of the positive patients of Group A $(18 ; 66.6 \%)$, resided in rural areas where L. infantum is considered to be autochthonous and $P$. perfiliewi is the predominant vector, as recently reported [8]. Theoretically, current global warming in all Mediterranean area may enhance leishmaniasis distribution due to the effect of temperature on parasite development and vector spread [7].

The high detection rate of circulant $L$. infantum DNA in a region where the pathogen is not considered endemic suggests that a significant local transmission of L. infantum to humans has been ongoing for years in these areas; in fact, Leishmania positivity in both study groups appears to be significantly related with residence in the rural rather than in urban context, highlighting the importance of a repeated and uninterrupted contact with the responsible vector and the animal reservoir [30].

So far, findings demonstrating higher rates of positivity in rural than in urban regions were recently reported in HIV positive patients in Morocco, where the risk of clinical VL is increased due to immunosuppression that may reactivate latent infections by L. infantum [31]. In other settings, travelling to an endemic area as well as the increasing international tourism and the influx of immigrants from endemic regions to non-endemic area can also be considered as significant risk factors for VL [32].

Four $(8 \%)$ out of 50 healthy controls from Group C resulted to be positive by qPCR. This result was not surprising in accordance with other reports [14, 33, 34]. As for Group A, the reasons for the recent expansion of VL cases in these areas are almost unknown.
The absence of DNA positivity in Group B is difficult to explain since data regarding subclinical infections in patients receiving steroid treatments are lacking. As reported, these therapies could cause reactivation of serious opportunistic infections in immunocompromised patients with lupus, pemphigus, transplant recipients [28] and may increase the risk for Leishmania-infected people to develop VL by affecting the effector, suppressor, and cytotoxic T cells functions through the blockade of cytokine expression, increasing susceptibility to intracellular pathogens, including Leishmania [35]. The absence of L. infantum circulant DNA in this group, despite the intake of steroid therapy, could be also due to the fact that only 19 (38\%) out of 50 patients resided in a rural context from some regional areas not fully involved by the circulation of the parasite and the competent vectors, with the consequence that less than half was exposed to Leishmania infection. This finding seems to support the hypothesis that only some regional areas are involved in the circulation of the parasite and the competent vector although the reasons for this phenomenon are not fully understood.

On the contrary, the detection of a very high parasitaemia in Group A, is an important finding that should be monitored as a potential indicator of a VL. BD therapies, by altering the inflammatory and immune balance, can impair the ability of host immune system to better control latent infections including leishmaniasis. Furthermore, it is likely that these patients did not develop clinical symptoms, despite the high level of parasitaemia and normal $\mathrm{CD}^{+}$lymphocyte count. In this regard, no change of T-helper and regulatory cells activity was observed also in cirrhotic patients with unusual fever from areas endemic for Leishmania, later diagnosed as VL after careful specific investigations [36].

In contrast to the Leishmania DNA parasitemia found in blood donors infected with Leishmania or in multiply transfused patients and in persons from highly endemic countries for $L$. infantum [37, 38], we are not aware of any data on Leishmania parasitemia in completely asymptomatic individuals taking BD in whom the host's immune response has not demonstrated to be changed or downregulated towards a Th- 2 cell-associated immune response against Leishmania. Probably, the exposure to rural areas appears to be a factor closely linked with the risk of developing Leishmania subclinical infection. In this regard, identification by qPCR of Leishmania DNA from asymptomatic cases, would allow new perspectives in Leishmania control and management. Although the presented data do not allow to make an interpretation on a parasitaemia dependent on the dose of the $\mathrm{BD}$, we believe that in absence of literature data in this setting, the most appropriate choice in these individuals is to monitor DNA parasitemia throughout the period of treatment with BD. Moreover, since incubation period of VL in patients treated with TNF-alpha antagonists is perhaps much longer than in non-immunosuppressed patients, as demonstrated in 
patients who have developed CL after such treatment [39], discontinuing TNF-a antagonist therapy should be done only when a diagnosis of leishmaniasis is established, and antileishmanial treatment should be started.

In our study, the decision to employ molecular methods for the diagnosis of asymptomatic Leishmania infection was supported by the fact that serology testing is quite specific and sensitive in diagnosing active VL in immunocompetent subjects [40, 41], although serological titers decline more or less rapidly in a significant portion of patients after the resolution of the disease [41]. Moreover, the above test frequently gives false-negative results in immunocompromised patients even in the presence of positive qPCR amplification [42] making their use unsatisfactory for detecting asymptomatic Leishmania infection, characterized by low or intermittent parasitaemia. Finally, the choice to extract and test DNA from PBMCs, instead of whole blood, has favoured the finding of high parasitic burdens, since PBMCs are target cells for the intracellular parasite [14].

Our data also showed that dog ownership was not correlated with Leishmania DNA positivity as domestic dogs play only a minor role in the transmission cycle of the parasite [43-45] even if the neighbour's dogs as well as the stray dogs present in large amounts in Northern Italy known to be highly seropositive for $L$. infantum [27], can be potential vectors of disease transmission. Moreover, rates of infection of sandflies fed with blood from VL patients are not dissimilar to those fed with infected dogs [43] and parasite prevalence in wild canines is often as high as in domestic dogs [45]. In this regard, to perform a canine serology near a patient's homes as a kind of sentinel for exposure to Leishmania would be of great interest.

With regard to the type of immunosuppressive treatment, we have not found statistically significant differences in relation to the BD administered (TNF- $\alpha$ antagonists, modulators of T lymphocyte activity and anti-IL-6 receptor antibodies) and to its possible influence on the molecular positivity for qPCR $\left(\chi^{2}(2)=3.37, p=0.185\right)$, although the percentage of the subjects positive to parasite DNA were higher when under therapy with modulators of $\mathrm{T}$ lymphocyte activity and anti-IL-6 receptor antibodies, compared to TNF- $\alpha$ antagonist treatment (Table 3, 57.1\%, 50.0\% and $26.3 \%$, respectively). This result, however, although interesting and worthy of further investigations, has not allowed to draw any statistical significance in this group for the small sample size.

Several literature data suggest that TNF- $\alpha$ antagonists may increase susceptibility to opportunistic infections, including leishmaniasis $[10,11,46]$.

Interestingly, the proportion of positive patients to $\mathrm{qPCR}$ (n. 18) statistically increases among those receiving the BD in combination with steroids (Table $3, \chi^{2}(1)=6.18, p<0.05$; 55.6 vs. $21.2 \%$ ). This finding supports several reports regarding cases of Leishmania reactivation in patients receiving chronic steroid treatment for different indications, including asthma, sarcoidosis, and myasthenia gravis [29].

Our study was limited by the small sample size and the lack of seroprevalence survey in property and neighbours' dogs. However, considering that Emilia-Romagna is a wellknown region with high canine seroprevalence for $L$. infantum (2\%, according data published in 2014) [47], living in a rural area or taking a steroid drug in addition to a biological therapy, were demonstrated (Table 4) to be factors strongly associated to an increased risk of being positive to Leishmania independently from sex and age [O.R. (95\% CI): 8.74 (2.24-34.05) and 5.52 (1.40-21.78), respectively]. This original finding seems to be relevant since current literature lacks sufficient data to fully explain the spread of leishmaniasis in endemic and hypo-endemic rural areas such as Emilia-Romagna region.

\section{Conclusion}

To our knowledge, the present study represents a first survey on Leishmania subclinical infection in rheumatologic patients receiving $\mathrm{BD}$ in an Italian region not traditionally considered endemic.

Since exposure to rural areas has been shown to be closely linked to the risk of developing a subclinical leishmanial infection that CIRD patients under BD seem to control less efficiently compared to healthy controls, screening and monitoring Leishmania DNA using molecular methods in candidates treated with BD living in these areas would be mandatory to prevent delay in diagnosis should VL symptoms appear.

Acknowledgements Mahmoud Rachid for his help in molecular methods; Gianluca Rugna for having provided Leishmania strains and Sara Bonazza for her help in collecting PBMC specimens.

Author contribution MM and CC equally contributed to the design of the study and the writing of the manuscript. MM CC and AT analysed and interpreted the patient's data. GM and AT performed the statistical analysis. PM, SA, SH and TB contributed to the revision of the article. MG contributed to the collection of patient's data. All authors read and approved the final version of the manuscript.

\section{Compliance with ethical standards}

Conflict of interest The authors declare that they have no competing interests.

Ethics approval The study was approved by the ethics committee of the University of Ferrara, Italy (protocol no. 150494).

Informed consent Written informed consents were obtained from all participants before their enrolment.

Consent for publication All the enrolled subjects had signed a written informed consent for publication. 
Data availability All the data included in the manuscript are available from the authors upon reasonable request.

\section{References}

1. Leishmaniasis - World Health Organization. Status Endemicity of cutaneous and visceral leishmaniasis worldwide. World Health Organization. 2018. http://www.who.int/news-room/fact-sheets/ detail/leishmaniasis.

2. Moral L, Rubio EM, Moya M. A leishmanian skin test survey in the human population of l' Alacantí region (Spain): implications for the epidemiology of Leishmania infantum infection in southern Europe. Trans R Soc Trop Med Hyg. 2002;96:129-32.

3. Poeppl W, Herkner H, Tobudic S, Faas A, Auer H, Mooseder G, et al. Seroprevalence and asymptomatic carriage of Leishmania spp. in Austria, a non-endemic European country. Clin Microbiol Infect. 2013;19:572-7.

4. Control of the leishmaniases: report of a meeting of the WHO Expert Commitee on the Control of Leishmaniases, Geneva, 22-26 March 2010. WHO technical report series 2010; n 949. Description. xiii, 1-186. http://apps.who.int/iris/bitstream/10665 /44412/1/WHO_TRS_949_eng.pdf.

5. Ready PD. Biology of phlebotomine sandflies as vectors of disease agents. Annu Rev Entomol. 2013;58:227-50.

6. Gradoni L. Pizzuti R, di Martino L, Gramiccia M, Pempinello R, Gaeta GB, Ferrara M, Scotti S, Altieri S. The epidemiology and surveillance of visceral leishmaniasis in the Campania region of Italy. The value of zymodeme typing. Epidemiol Infect. 1993;111:297-306.

7. Varani S, Cagarelli R, Melchionda F, Attard L, Salvadori C, Finarelli AC, Gentilomi GA, et al. Ongoing outbreak of visceral leishmaniasis in Bologna Province, Italy, November 2012 to May 2013. Euro Surveill. 2013;18:20530.

8. Leishmaniasis. Fact sheet. World Health Organization. Media Centre. http://www.who.int/mediacentre/factsheet/fs375/en/. Accessed Sep 2016

9. Xynos ID, Tektonidou MG, Pikazis D, Sipsas NV. Leishmaniasis, autoimmune rheumatic disease, and anti-tumor necrosis factor therapy, Europe. Emerg Infect Dis. 2009;15:956-9.

10. Zanger P, Kotter I, Kremsner PG, Gabrysch S. Tumor necrosis factor alpha antagonist drugs and leishmaniasis in Europe. Clin Microbiol Infect. 2012;18:670-6.

11. Martin-Mola E, Balsa A. Infectious complications of biologic agents. Rheum Dis Clin North Am. 2009;35:183-99.

12. Srivastava P, Gidwani K, Picado A, Van der Auwera G, Tiwary $\mathrm{P}$, et al. Molecular and serological markers of Leishmania donovani infection in healthy individuals from endemic areas of Bihar, India. Trop Med Int Health. 2013;18:548-54.

13. Sakkas H, Gartzonika C, Levidiotou S. Laboratory diagnosis of human visceral leishmaniasis. J Vector Borne Dis. 2016;53:8-16.

14. le Fichoux Y, Quaranta JF, Aufeuvre JP, Lelievre A, Marty P, Suffia I, et al. Occurrence of Leishmania infantum parasitemia in asymptomatic blood donors living in an area of endemicity in southern France. J Clin Microbiol. 1999;37:1953-7.

15. Riera C, Fisa R, Udina M, Gállego M, Portus M. Detection of Leishmania infantum cryptic infection in asymptomatic blood donors living in an endemic area (Eivissa, Balearic Islands, Spain) by different diagnostic methods. Trans R Soc Trop Med Hyg. 2004;98:102-10.

16. Colomba C, Saporito L, Vitale F, Reale S, Vitale G, Casuccio A, et al. Cryptic Leishmania infantum infection in Italian HIV infected patients. BMC Infect Dis. 2009;9:199.
17. Maritati M, Michel G, Almugadam S, Hanau S, Bellini T, Dallocchio F, et al. Real-time PCR to detect Leishmania Infantum DNA in immunocompetent patients with chronic inflammatory rheumatisms living in rural areas of Northern Italy and treated with biologic drugs (oral communication 0328). 26th ESCMID, Amsterdam, The Netherlands, 9-12 April 2016. ESCMID Library O328.

18. Contini C, Grilli A, Badia L, Guardigni V, Govoni M, Seraceni S. Detection of Chlamydophila pneumoniae in patients with arthritis: significance and diagnostic value. Rheumatol Int. 2011;31:1307-13.

19. McCarthy-Burke C, Bates PA, Dwyer DM. Leishmania donovani: use of two different, commercially available, chemically defined media for the continuous in vitro cultivation of promastigotes. Exp Parasitol. 1991;73:385-7.

20. Lachaud L, Chabbert E, Dubessay P, Dereure J, Lamothe J, Dedet JP, Bastien P. Value of two PCR methods for the diagnosis of canine visceral leishmaniasis and the detection of asymptomatic carriers. Parasitology. 2002;125:197-207.

21. Dujardin JC, Campino L, Cañavate C, Dedet J-P, Gradoni L, Soteriadou K, et al. Spread of vector-borne diseases and neglect of Leishmaniasis, Europe. Emerg Infect Dis. 2008;14:1013-18.

22. Mary C, Faraut F, Lascombe L, Dumon H. Quantification of Leishmania infantum DNA by a real-time PCR assay with high sensitivity. J Clin Microbiol. 2004;42:5249-55.

23. Di Muccio T, Scalone A, Bruno A, Marangi M, Grande R, Armignacco O, Gradoni L, Gramiccia M. Epidemiology of imported leishmaniasis in Italy: implications for a European Endemic Country. Plos ONE. 2015;10:e0129418.

24. Okwor I, Uzonna JE. The immunology of Leishmania/HIV coinfection. Immunol Res. 2013;56:163-71.

25. Maroli M, Feliciangeli MD, Bichaud L, Charrel RN, Gradoni L. Phlebotomine sandflies and the spreading of leishmaniases and other diseases of public health concern. Med Vet Entomol. 2013;27:123-47.

26. Signorini M, Cassini R, Drigo M, Frangipane di Regalbono A, Pietrobelli M, Montarsi F, Stensgaard AS. Ecological niche model of Phlebotomus perniciosus, the main vector of canine leishmaniasis in north-eastern Italy. Geospat Health. 2014;9:193-201.

27. Maroli M, Rossi L, Baldelli R, Capelli G, Ferroglio E, Genchi C, Gramiccia M, Mortarino M, Pietrobelli M, Gradoni L. The northward spread of leishmaniasis in Italy: evidence from retrospective and on-going studies on the canine reservoir and phlebotomine vectors. Trop Med Int Health. 2008;13:256-64.

28. van Griensven J, Carrillo E, Lopez-Velez R, Lynen L, Moreno J. Leishmaniasis in immunosuppressed individuals. Clin Microbiol Infect. 2014;20:286-99.

29. Marie I, Guglielmino E. Non tuberculous anti-TNF associated opportunistic infections. Rev Med Interne. 2010;31(5):353-60.

30. Biglino A, Bolla C, Concialdi E, Trisciuoglio A, Romano A, Ferroglio E. Asymptomatic Leishmania infantum infection in an area of North Western Italy (Piedmont region) where such Infections are traditionally nonendemic. J Clin Microbiol. 2010;48:131-6.

31. Echchakery M, Nieto J, Boussaa S, El Fajali N, Ortega S, Souhail $\mathrm{K}$, Aajly H, Chicharro C, Carrillo E, Moreno J, Boumezzough A. Asymptomatic carriers of Leishmania infantum in patients infected with human immunodeficiency virus (HIV) in Morocco. Parasitol Res. 2018;117:1237-44.

32. Mansueto P, Seidita A, Vitale G, Cascio A. Leishmaniasis in travelers: a literature review. Travel Med Infect Dis. 2014;12:563-81.

33. Marty P, Lelievre A, Quaranta JF, Rahal A, Gari-Toussaint M, Le Fichoux Y. Use of the leishmanin skin test and western blot analysis for epidemiological studies in visceral leishmaniasis areas: experience in a highly endemic focus in Alpes-Maritimes (France). Trans R Soc Trop Med Hyg. 1994;88:658-9. 
34. Colomba C, Saporito L, Polara VF, Barone T, Corrao A, Titone L. Serological screening for Leishmania infantum in asymptomatic blood donors living in an endemic area (Sicily, Italy). Transfus Apher Sci. 2005;33:311-4.

35. Pittalis S, Nicastri E, Spinazzola F, Ghirga P, De Marco M, Paglia MG, Narciso P. Leishmania infantum leishmaniasis in corticosteroid-treated patients. BMC Infect Dis. 2006;18:177.

36. Pagliano P, Carannante N, Gramiccia M, Ascione T, Stornaiuolo G, Gradoni L, Faella FS, Gaeta GB. Visceral leishmaniasis causes fever and decompensation in patients with cirrhosis. Gut. 2007;56:893-4.

37. Tordini G, Puttini C, Rossetti B, Sammarro G, Fanetti A, Cianchino $\mathrm{S}$, et al. Which screening for Leishmania infantum in asymptomatic blood donors? Infez Med. 2011;19:152-6.

38. Jimenez-Marco T, Riera C, Girona-Llobera E, Guillen C, Iniesta $\mathrm{L}$, Alcover M, et al. Strategies for reducing the risk of transfusion-transmitted leishmaniasis in an area endemic for Leishmania infantum: a patient- and donor-targeted approach. Blood Transfus. 2018;16:130-136. https://doi.org/10.2450/2017.0201-16. Epub 2017 Mar 15.

39. Neumayr AL, Morizot G, Visser LG, Lockwood DN, Beck BR, Schneider S, Bellaud G, Cordoliani F, Foulet F, Laffitte EA, Buffet $\mathrm{P}$, Blum JA. Clinical aspects and management of cutaneous leishmaniasis in rheumatoid patients treated with TNF- $\alpha$ antagonists. Travel Med Infect Dis. 2013;11:412-20. https://doi.org/10.1016/j. tmaid.2013.05.003. (epub 2013 Aug 22).

40. Kumar R, Pai K, Pathak K, Sundar S. Enzyme-linked immunosorbent assay for recombinant $\mathrm{K} 39$ antigen in diagnosis and prognosis of Indian visceral leishmaniasis. Clin Diagn Lab Immunol. 2001;8:1220-24.
41. De Almeida Silva L, Romero HD, Prata A, Costa RT, Nascimento E, Carvalho SF, Rodrigues V. Immunologic tests in patients after clinical cure of visceral leishmaniasis. Am J Trop Med Hyg. 2006;75:739-43.

42. Garcia-Garcia A, Martin-Sanchez J, Gallego M, Rivero-Roman A, Camacho A, Riera C, Morillas-Marquez F, Vergara S, Macias J, Pineda JA. Use of noninvasive markers to detect Leishmania infection in asymptomatic human immunodeficiency virusinfected patients. J Clin Microbiol. 2006;44:4455-58.

43. Costa CHN, Pereira HF, Pereira FCA, Tavares JP, Araújo MV, Gonçalves MJO. Is the household dog a risk factor for American visceral leishmaniasis in Brazil. Trans R Soc Trop Med Hyg. 1999;93:464.

44. Costa CHN, Gomes RBB, Silva MRB, Garcez LM, Ramos PKS, Santos RS, Shaw JJ, David JR, Maguire JH. Competence of the human host as a reservoir for Leishmania chagasi. J Infect Dis. 2000;182:997-1000.

45. Gavgani ASM, Mohite H, Edrissian GH, Mohebali M, Clive $\mathrm{RD}$. Domestic dog ownership in Iran is a risk factor for human infection with Leishmania infantum. Am J Trop Med Hyg. 2002;67:511-15.

46. Jeziorski E, Dereure J, Mac Bullen G, Blanchet C, Ludwig C, Costes V, Rodière M. Mucosal relapse of visceral leishmaniasis in a child treated with anti-TNF $\alpha$. Int J Infect Dis. 2015;33:135-6.

47. Regione Emilia-Romagna: Progetto regionale di sorveglianza sulle malattie trasmesse da insetti vettori. Piano Regionale di controllo della leishmaniosi - anno 2014. http://www.anagrafeca ninarer.it/acrer/Portals/0/normative/Relazione_Piano_Leishmania _2014\%20senza\%20tabelle\%20canili.pdf. 